Pacific Journal of Mathematics

GENERATING MONOMIALS FOR FINITE SEMIGROUPS 


\title{
GENERATING MONOMIALS FOR FINITE SEMIGROUPS
}

\author{
DONALD C. RAMSEY
}

In this paper consideration is given semigroups which arise from a group $(G, \cdot)$ by defining a binary operation o on $G$ by the rule

$$
x \circ y=x \phi y \phi \quad \text { for all } x, y \text { in } G \text {, }
$$

where $\phi, \phi$ are endomorphisms of $G$. In particular, the structure of such semigroups is determined. Also determined are the structure and number of semigroups that can be defined by

$$
x \circ y=a x^{s} y^{t} \quad \text { for all } x, y \text { in } G \text {, }
$$

where $(G, \cdot)$ is a finite abelian group containing $a$, and $s, t$ are nonnegative integers.

1. Introduction. Let $(G, \cdot)$ be a groupoid and let $\phi, \psi$ be transformations of $G$. A possibly different groupoid $(G, \circ)$ is defined by the rule

$$
x \circ y=x \phi y \psi \quad \text { for all } x, y \text { in } G \text {. }
$$

In $\S 2$ of this paper we assume that $(G, \cdot)$ is a finite abelian group and define a groupoid $(G, \circ)$ by the rule

$$
x \circ y=a x^{s} y^{t} \quad \text { for all } x, y \text { in } G \text {, }
$$

where $s, t$ are nonnegative integers and $a \in G$. Necessary and sufficient conditions on $a, s$, and $t$ are found in order for $(G, \circ)$ to be a semigroup. Also, we determine the number of nonequivalent (i.e., non-isomorphic, non-anti-isomorphic) semigroups that are defined in this manner. Whenever the rule

$$
x \circ y=a x^{s} y^{t} \quad \text { for all } x, y \text { in } G,
$$

defines a semigroup, we say that $(G, \circ)$ is generated by the monomial $a x^{s} y^{t}$ over $(G, \cdot)$.

In $\S 3$ it is shown that if a semigroup $(G, \circ)$ is defined by the rule

$$
x \circ y=x \phi y \psi \quad \text { for all } x, y \text { in } G,
$$

where $\dot{\phi}, \psi$ are endomorphisms of the group $(G, \cdot)$, then $(G, \circ)$ is an inflation of the direct product of a group and a rectangular band. Consequently, a semigroup generated by a monomial over a finite abelian group is an inflation of the direct product of a group 
and a rectangular band. Finally, if $\left(F_{q},+, \cdot\right)$ is a finite field of order $q$ and if the rule

$$
x \circ y=a x^{s} y^{t} \quad \text { for all } x, y \text { in } F_{q},
$$

where $a \in F_{q}$ defines a semigroup $\left(F_{q}, \circ\right)$, then $\left(F_{q}, \circ\right)$ is an inflation of the direct product of a cyclic group and a rectangular band, together with a zero element. This is a generalization of the results obtained in [3] by Plemmons and Yoshida.

2. Generating monomials. Throughout this section let $(G, \cdot)$ be a finite abelian group with identity element $e$, and let $M$ denote the least common multiple of the orders of the elements of $G$. Then $M$ is the least positive integer $q$ such that $x^{q}=e$ for all $x$ in $G$. The following theorem gives necessary and sufficient conditions on a monomial $a x^{s} y^{t}$ over $(G, \cdot)$, in order for it to generate a semigroup.

THEOREM 1. The monomial ax $y^{t}$ generates a semigroup over $(G, \cdot)$ if and only if

(i) $a^{s-t}=e$ and

(ii) $s^{2}-s$ and $t^{2}-t$ are multiples of $M$.

Proof. The monomial $a x^{s} y^{t}$ generates a semigroup over $(G, \cdot)$ if and only if for all $x, y, z$ in $G$

$$
a\left(a x^{s} y^{t}\right)^{s} z^{t}=a x^{s}\left(a y^{s} z^{t}\right)^{t}
$$

which holds if and only if for all $x, y, z$ in $G$

$$
a^{s+1} x^{s^{2}} y^{s t} z^{t}=a^{t+1} x^{s} y^{s t} z^{t^{2}}
$$

which in turn holds if and only if for all $x, z$ in $G$

$$
a^{s-t} x^{s^{2}-s}=z^{t^{2}-t} .
$$

Assuming that (i) and (ii) hold, it follows that (2.1) holds since each side of the equation reduces to $e$. Thus $a x^{s} y^{t}$ generates a semigroup. Conversely, if $a x^{s} y^{t}$ generates a semigroup then equation (2.1) holds for all $x, z$ in $G$, and in particular when $x=z=e$, so that $a^{s-t}=e$. By letting $z=e$ in equation (2.1) and replacing $a^{s-t}$ by $e$, we get that $x^{s^{2}-s}=e$ for all $x$ in $G$, whence $s^{2}-s$ is a multiple of $M$. In a similar fashion it can be shown that $t^{2}-t$ is a multiple of $M$.

If $s \geqq M$, then $s=q M+r$ for some integers $q$ and $r$, where $q>0$ and $0 \leqq r<M$, so that

$$
a x^{s} y^{t}=a x^{r} y^{t} \quad \text { for all } x, y \text { in } G \text {. }
$$


Hence, in searching for the number of nonequivalent semigroups generated by monomials over $(G, \cdot)$ we can assume that $0 \leqq s<M$ and $0 \leqq t<M$. Also, since the semigroup generated by $a x^{t} y^{s}$ is anti-isomorphic to the one generated by $a x^{s} y^{t}$ we can assume that $t \leqq s$. Furthermore, the following lemma shows that we need only consider monomials with $a=e$.

Lemma 1. Suppose ax $x^{s} y^{t}$ generates a semigroup $(G, \circ)$ over $(G, \cdot)$. Let $(G, *)$ be the semigroup generated by $x^{s} y^{t}$ and let $k$ denote the order of $a$ in $(G, \cdot)$. Let $m$ be the solution to the congruence

$$
(2 t-1) x \equiv 1 \quad(\bmod k) .
$$

Then $m$ is unique $(\bmod k)$ and the mapping $\alpha$ from $G$ into $G$ defined by

$$
x \alpha=a^{m} x \quad \text { for all } x \text { in } G,
$$

is an isomorphism of $(G, \circ)$ onto $(G, *)$.

Proof. Since $k$ is the order of $a$ in $(G, \cdot)$, it follows that $k \mid M$. Since $a x^{s} y^{t}$ generates a semigroup, $M \mid t^{2}-t$, whence $k \mid t^{2}-t$. Therefore, the greatest common divisor of $2 t-1$ and $k$ must divide $(2 t-1)^{2}-4\left(t^{2}-t\right)=1$, whence $2 t-1$ and $k$ are relatively prime. Hence [2, Theorem 3-11, p. 34] there exists a unique solution $m$ $(\bmod k)$ to the congruence

$$
(2 t-1) x \equiv 1 \quad(\bmod k) .
$$

Therefore $k$ is a factor of $m(2 t-1)-1$. Now, the mapping $\alpha$ from $G$ into $G$ defined by

$$
\alpha: z \rightarrow a^{m} z
$$

is a permutation of $G$. Let $x, y$ be arbitrary elements of $G$. Then

$$
\begin{aligned}
(x \alpha) *(y \alpha) & =\left(a^{m} x\right)^{s}\left(a^{m} y\right)^{t} \\
& =a^{m(s+t)} x^{s} y^{t} \\
& =a^{m+1} x^{s} y^{t}
\end{aligned}
$$

since

$$
a^{m(s+t)-(m+1)}=a^{m(s+t-1)-1}=a^{m(s-t)+m(2 t-1)-1}=e .
$$

Therefore,

$$
\begin{aligned}
(x \alpha) *(y \alpha) & =a^{m+1} x^{s} y^{t} \\
& =\left(a x^{s} y^{t}\right) \alpha \\
& =(x \circ y) \alpha .
\end{aligned}
$$


Thus $\alpha$ is an isomorphism of $(G, \circ)$ onto $(G, *)$.

Let $n$ denote the order of $(G, \cdot)$ and let $n=p_{1}^{\alpha_{1}} p_{2}^{\alpha_{2}} \cdots p_{r}^{\alpha_{r}}$ be the prime power factorization of $n$, where $p_{i} \neq p_{j}$ if $i \neq j$, and $\alpha_{i}>0$ for $1 \leqq i \leqq r$. By the fundamental theorem for finite abelian groups, $G$ has the structure $S\left(p_{1}\right) \times S\left(p_{2}\right) \times \cdots \times S\left(p_{r}\right)$ where each $S\left(p_{i}\right)$ is the Sylow $p$-subgroup of $(G, \cdot)$ of order $p_{i}^{\alpha}$ for $1 \leqq i \leqq r$. The order of any element in $S\left(p_{i}\right)$ is a power of the prime $p_{i}$ so that for each prime $p_{i}$ with $1 \leqq i \leqq r$, there exists an element $x_{i} \in G$ having order a power $>0$ of $p_{i}$. Thus the prime power factorization of $M$ is $M=p_{1}^{\gamma_{1}} p_{2}^{\gamma_{2}} \cdots p_{r}^{\gamma_{r}}$ where $0<\gamma_{i} \leqq \alpha_{i}$ for $1 \leqq i \leqq r$.

For each integer $m$ let

$$
G_{m}=\left\{x \in G: x^{m}=e\right\} .
$$

Let $s$ be a positive integer such that $M \mid s(s-1)$. Since $s$ and $s-1$ are relatively prime, the prime factors of $M$ which divide $s$ do not divide $s-1$, and those dividing $s-1$ do not divide $s$. Assume that the indexing of the primes $p_{i}$ in the factorization of $M$ is such that $p_{1}^{\gamma_{1}} p_{2}^{\gamma_{2}} \cdots p_{j}^{\gamma j} \mid(s-1)$ and $p_{j+1}^{\gamma_{j+1}} p_{j+2}^{\gamma_{j+2}} \cdots p_{r}^{\gamma} \mid s$. Identifying the elements of $G$ and $S\left(p_{1}\right) \times S\left(p_{2}\right) \times \cdots \times S\left(p_{r}\right)$ we get the following lemma.

LEMma 2. The set $G_{s-1}$ is the subgroup $S\left(p_{1}\right) \times S\left(p_{2}\right) \times \cdots S\left(p_{j}\right)$ of $G$ having order $p_{1}^{\alpha_{1}} p_{2}^{\alpha_{2}} \cdots p_{j}^{\alpha_{j}}$.

Proof. Let $x \in G_{s-1}$. Written as an $r$-tuple, $x=\left(x_{1}, x_{2}, \cdots, x_{r}\right)$, so $x^{s-1}=\left(x_{1}^{s-1}, x_{2}^{s-1}, \cdots, x_{r}^{s-1}\right)=e_{r}$, where $e_{r}$ is the $r$-tuple $(e, e, \cdots, e)$. In particular, $x_{j+1}^{s-1}=x_{j+2}^{s-1}=\cdots=x_{r}^{s-1}=e$. Since the orders of $x_{j+1}, x_{j+2}, \cdots, x_{r}$ are relatively prime to $s-1$ it follows that $x_{j+1}=$ $x_{j+2}=\cdots=x_{r}=e$. Hence $x \in S\left(p_{1}\right) \times S\left(p_{2}\right) \times \cdots \times S\left(p_{j}\right)$. Conversely, let $x \in S\left(p_{1}\right) \times S\left(p_{2}\right) \times \cdots \times S\left(p_{j}\right)$. We write

$$
x=\left(x_{1}, x_{2}, \cdots, x_{j}\right) \text {. }
$$

Letting $e_{j}$ denote the $j$-tuple $(e, e, \cdots, e)$, we have

$$
e_{j}=x^{s(s-1)}=\left(x_{1}^{s(s-1)}, x_{2}^{s(s-1)}, \cdots, x_{j}^{s(s-1)}\right),
$$

so that $x_{1}^{s(s-1)}=x_{2}^{s(s-1)}=\cdots=x_{j}^{s(s-1)}=e$. Since the orders of $x_{1}, x_{2}, \cdots, x_{j}$ are relatively prime to $s, x_{1}^{s-1}=x_{2}^{s-1}=\cdots=x_{j}^{s-1}=e$, whence $x^{s-1}=e_{j}$ and $x \in G_{s-1}$.

Lemma 3. Let $s$ and $s^{\prime}$ be positive integers less than $M$ such that $M \mid s^{2}-s$ and $M \mid s^{\prime 2}-s^{\prime}$. If the order of $G_{s-1}$ is the same as the order of $G_{s^{\prime}-1}$ then $s=s^{\prime}$. 
Proof. By Lemma 2 the subgroups $G_{s-1}$ and $G_{s^{\prime}-1}$ are direct products of Sylow $p$-subgroups of $G$. Since the order of $G_{s-1}$ is the same as the order of $G_{s^{\prime}-1}$, it follows that the prime powers in the factorization of $M$ which divide $s-1$ are exactly those which divide $s^{\prime}-1$. Thus $M \mid s\left(s^{\prime}-1\right)$ and $M \mid s^{\prime}(s-1)$, whence

$$
M \mid\left[s\left(s^{\prime}-1\right)-s^{\prime}(s-1)\right],
$$

so $M \mid s^{\prime}-s$. Since $-M<s^{\prime}-s<M, s^{\prime}-s=0$, whence $s^{\prime}=s$.

THEOREM 2. Suppose $x^{s} y^{t}$ and $x^{s^{\prime}} y^{t^{\prime}}$ generate semigroups over $(G, \cdot)$, where $0 \leqq t \leqq s<M$ and $0 \leqq t^{\prime} \leqq s^{\prime}<M$. Then these semigroups are isomorphic if and only if $s=s^{\prime}$ and $t=t^{\prime}$.

Proof. Clearly if $s=s^{\prime}$ and $t=t^{\prime}$ then $x^{s} y^{t}$ and $x^{s^{\prime}} y^{t^{\prime}}$ generate the same semigroup over $(G, \cdot)$. Conversely, suppose that $x^{s} y^{t}$ and $x^{s^{\prime}} y^{t^{\prime}}$ generate semigroups $(G, \circ)$ and $(G, *)$, respectively, and suppose $(G, \circ)$ is isomorphic to $(G, *)$. Then the Cayley tables for $(G, \circ)$ and $(G, *)$ must have the same number of distinct rows. That is, $(G, \circ)$ and $(G, *)$ must have the same number of distinct inner left translations [1, p. 9]. The distinct inner left translations of $(G, \circ)$ are determined by the distinct elements of the set $\left\{x^{s}: x \in G\right\}$. But

$$
\left\{x^{s}: x \in G\right\}=G_{s-1}
$$

as defined above. Thus the orders of $G_{s-1}$ and $G_{s^{\prime}-1}$ are equal, whence by Lemma $3, s=s^{\prime}$ if both $s$ and $s^{\prime}$ are positive. If $s=0$ then $G_{s^{\prime}-1}=G_{s-1}=\{e\}$, so that $M \mid s^{\prime}$, whence $s^{\prime}=0$. Similarly, if $s^{\prime}=0$ then $s=0$, so that in any case $s=s^{\prime}$. Dually, by considering columns in the Cayley tables of $(G, \circ)$ and $(G, *)$, we see that $t=t^{\prime}$.

We now approach the problem of determining the number of nonequivalent semigroups of order $n$ generated by monomials over $(G, \cdot)$. The integers $s$ with $0 \leqq s<M$ that will serve as exponents in generating monomials are exactly those such that $M \mid s^{2}-s$. Hence the set $H$ of such integers is the solution set of the congruence

$$
x^{2}-x \equiv 0 \quad(\bmod M) \text {. }
$$

LEMma 4. The cardinality of the solution set $H$ to the congruence (2.2) is $2^{r}$, where $r$ is the number of distinct primes in the prime power factorization of $M$.

Proof. Let $M=p_{1}^{\gamma_{1}} p_{2}^{\gamma_{2}} \cdots p_{r}^{r}$ be the prime power factorization of $M$. Then $x_{0}$ is a solution to (2.2) if and only if $x_{0}$ is a simultaneous solution to the system of congruences 


$$
x^{2}-x \equiv 0 \quad\left(\bmod p_{i}^{\gamma} i\right) \quad 1 \leqq i \leqq r .
$$

For each $i, 1 \leqq i \leqq r$, suppose $c_{i}$ is a solution to $x^{2}-x \equiv 0\left(\bmod p_{i}^{\gamma}\right)$. Then, by the Chinese Remainder Theorem, there is a solution $x_{0}$ to the system

$$
x \equiv c_{1}\left(\bmod p_{1}^{\gamma_{1}}\right), x \equiv c_{2}\left(\bmod p_{2}^{\gamma_{2}}\right), \cdots, x \equiv c_{r}\left(\bmod p_{r}^{\gamma_{r}}\right)
$$

which is unique modulo $M$. Then each $r$-tuple $\left(c_{1}, \cdots, c_{r}\right)$ gives rise to a unique solution $(\bmod M)$ to system (2.3). Thus the number of solutions to (2.2) is the product of the numbers of roots of the congruences in (2.3). But, by $\S 3.5$ of [2], the solution set to each of these congruences is $\{0,1\}$, whence the cardinality of the solution set of (2.2) is $2^{r}$.

Finally, we have the following theorem.

THEOREM 3. The number $N_{G}$ of nonequivalent semigroups generated by monomials over $(G, \cdot)$ is $2^{r-1}\left(2^{r}+1\right)$, where $r$ is the number of distinct primes which divide $M$.

Proof. The pairs $s, t$ of elements of $H$ yield monomials $x^{s} y^{t}$ which generate semigroups over $(G, \cdot)$. Moreover, these are the only pairs modulo $M$ which will do so. Thus to determine $N_{G}$ we need only count the ways in which $s$ and $t$ can be picked from $H$ with $t \leqq s$. There are

$$
1+2+3+\cdots+2^{r}=\frac{2^{r}\left(2^{r}+1\right)}{2}=2^{r-1}\left(2^{r}+1\right)
$$

ways to do this.

3. Structure theorems. The following definition and facts are the contents of $[1$, p. 98, Exercise 10]. Let $T$ be a semigroup. With each element $\alpha$ of $T$, associate a set $X_{\alpha}$ containing $\alpha$ such that the sets $X_{\alpha}$ are mutually disjoint. Let $s=\mathrm{U}_{\alpha \in T} X_{\alpha}$, and let the product in $T$ be extended to a product in $S$ by defining $a b=\alpha \beta$ if $a \in X_{\alpha}$ and $b \in X_{\beta}$. Then $S$ is a semigroup and is said to be an inflation of $T$. Now, $T$ is a subsemigroup of $S$ such that $S^{2} \subseteq T$. If we define a mapping $\theta$ from $S$ into $T$ by $a \theta=\alpha$ when $a \in X_{\alpha}$, then

(i) $\theta$ maps $S$ upon $T$,

(ii) $\theta^{2}=\theta$, and

(iii) $(a \theta)(b \theta)=a b \quad$ for all $a, b \in S$.

Let $T$ be a subsemigroup of $S$ such that $S^{2} \subseteq T$, and let $\theta$ be a transformation of $S$ having properties (i), (ii), and (iii) above. Then $S$ is an inflation of $T$.

By a left zero semigroup we mean a semigroup $S$ such that $x y=x$ 
for all $x, y \in S$. A right zero semigroup is defined dually.

THEOREM 4. Let $(S, \cdot)$ be a semigroup such that for some transformation $\phi$ of $S, x y=x \phi$ for all $x, y \in S$. Then $S$ is an inflation of the range $S \phi$ of $\phi$, and $S \phi$ is a left zero semigroup. Conversely, each inflation of a left zero semigroup is obtained in this manner.

Proof. Since $S$ is a semigroup, $(x y) z=x(y z)$ for all $x, y, z \in S$, so $x \phi^{2}=x \phi$ for all $x \in S$, whence $\phi^{2}=\phi$ on $S$. Since $S^{2}=S \phi, S \phi$ is a subsemigroup of $S$ such that $S^{2} \cong S \phi$. Now $\phi$ maps $S$ onto $S \phi$ and

$$
a \phi b \phi=a \phi^{2}=a \phi=a b \quad \text { for all } a, b \in S .
$$

Hence, $S$ is an inflation of $S \phi$. Let $a, b \in S \phi$. Then $a=a \phi$, so

$$
a b=a \phi b=a \dot{\phi}^{2}=a \phi=a,
$$

thus $S_{\phi}$ is a left zero semigroup. Conversely, let $(S, \cdot)$ be an inflation of a left zero semigroup $L$. Since $S$ is an inflation of $L, S$ is the disjoint union of subsets $X_{a}$, where $a \in L \cap X_{a}$. Define a transformation $\phi$ of $S$ by $x \phi=a$ if and only if $x \in X_{a}$. Let $x, y \in S$ with $x \in X_{a}$ and $y \in X_{b}$. Then $x y=a b=a=x \phi$.

COROLLARY 1. If $(G, \circ)$ is generated by $x^{s}$ over a finite abelian group $(G, \cdot)$, then $(G, \circ)$ is an inflation of the left zero semigroup $(L, \circ)$, where $L=\left\{x^{s}: x \in G\right\}$.

By the dual of Theorem 4 we get the following corollary.

COROLlaRY 2. If $(G, \circ)$ is generated by $y^{t}$ over the finite abelian group $(G, \cdot)$, then $(G, \circ)$ is an inflation of the right zero semigroup $(R, \circ)$, where $R=\left\{y^{t}: y \in G\right\}$.

Before investigating the structure of semigroups generated by the more general monomial $x^{s} y^{t}$ with $0 \leqq t \leqq s<M$, we prove the following lemma.

Lemma 5. Suppose the semigroup $(G, \circ)$ is generated by $x^{s} y^{t}$ over an abelian group $(G, \cdot)$ with $0 \leqq t \leqq s<M$. Then $\circ$ is commutative if and only if $s=t$.

Proof. Suppose $s=t$. Then for $x, y \in G$ we have

$$
x \circ y=x^{s} y^{s}=y^{s} x^{s}=y \circ x .
$$


Conversely, if $\circ$ is commutative, then $x \circ y=y \circ x$ for all $x, y \in G$, so that $x^{s} y^{t}=y^{s} x^{t}$ for all $x, y \in G$. Letting $y=e$, we see that $x^{s}=x^{t}$ for all $x \in G$, so that $M \mid s-t$. Thus $s-t=0$, whence $s=t$.

Given an arbitrary group $(G, \cdot)$ and a pair of transformations $\phi$, $\psi$ of $G$, a groupoid $(G, \circ)$ is defined by the rule

$$
x \circ y=x \phi y \psi \quad \text { for all } x, y \text { in } G \text {. }
$$

We say that $(G, \circ)$ is generated by the pair of transformations $(\phi, \psi)$ over $(G, \cdot)$. If we insist that the transformations $\phi$ and $\psi$ be endomorphisms, the following lemma gives necessary and sufficient conditions in order for $(G, \circ)$ to be a semigroup.

LemMa 6. Let $(G, \cdot)$ be an arbitrary group with identity element $e$, and let $\phi, \psi$ be endomorphisms of $(G, \cdot)$. Define a groupoid $(G, \circ)$ by the rule

$$
x \circ y=x \phi y \psi \quad \text { for all } x, y \text { in } G .
$$

Then $(G, \circ)$ is a semigroup if and only if $\phi$ and $\psi$ are idempotent and commute.

Proof. Assume that the groupoid $(G, \circ)$ is a semigroup. Then

$$
(x \circ y) \circ z=x \circ(y \circ z) \quad \text { for all } x, y, z \text { in } G \text {, }
$$

so

$$
(x \phi y \psi) \dot{\phi} \cdot z \psi=x \phi(y \phi z \psi) \psi \quad \text { for all } x, y, z \text { in } G .
$$

Upon setting $y=z=e$ in (3.1), we get

$$
(x \phi) \phi=x \phi \quad \text { for all } x \text { in } G,
$$

since $e \phi=e \psi=e$. In a similar fashion $\psi^{2}=\psi$. Letting $x=z=e$ in (3.1), we see that

$$
(y \psi) \phi=(y \dot{\phi}) \psi \quad \text { for all } y \text { in } G,
$$

hence $\phi \psi=\psi \phi$. Conversely, assume that $\phi^{2}=\phi, \psi^{2}=\psi$, and $\phi \psi=$ $\psi \phi$. Then for arbitrary $x, y, z \in G$

$$
\begin{aligned}
(x \circ y) \circ z & =(x \phi y \psi) \phi \cdot z \psi \\
& =x \phi^{2} \cdot y \psi \phi \cdot z \psi \\
& =x \phi \cdot y \phi \psi \cdot z \psi^{2} \\
& =x \phi(y \phi z \psi) \psi \\
& =x \circ(y \circ z) .
\end{aligned}
$$

Thus $(G, \circ)$ is a semigroup. 
The following definitions come from [1, p. 98, p. 25]. A semigroup $S$ is called stationary on the right if for all $a, b, c$ in $S, a b=a c$ implies $x b=x c$ for all $x \in S$. A semigroup $S$ is called $E$-inversive if for each $a \in S$ there exists $x \in S$ such that $a x$ is idempotent. Let $a, b, x$, $y$ be elements of a semigroup $S$. Consider the four elements $a x, a y$, $b x$, by of $S$. We call $S$ rectangular if, whenever three elements are equal, all four are equal. Let $X$ and $Y$ be any two sets, and define a binary operation in $S=X \times Y$ by

$$
\left(x_{1}, y_{1}\right)\left(x_{2}, y_{2}\right)=\left(x_{1}, y_{2}\right)
$$

where $x_{1}, x_{2} \in X$ and $y_{1}, y_{2} \in Y$. Then $S$ is a semigroup called the rectangular band on $X \times Y$.

THEOREM 5. Let $(G, \circ)$ be a semigroup generated by a pair of endomorphisms $(\phi, \psi)$ over the group $(G, \cdot)$. Then $(G, \circ)$ is an inflation of its kernel $G \circ G$ and its kernel is isomorphic to the direct product of a group and a rectangular band.

Proof. By Lemma 6, $\phi^{2}=\phi, \psi^{2}=\psi$, and $\phi \psi=\psi \phi$. Now $(G, \circ)$ is stationary on the right, since if $a \circ b=a \circ c$ for arbitrary $a, b$, $c \in G$ then $a \phi b \psi=a \phi c \psi$, so $b \psi=c \psi$. Thus $x \phi b \psi=x \phi c \psi$ for all $x \in G$, so that $x \circ b=x \circ c$ for all $x \in G$. Let $a \in G$ and denote by $a^{-1}$ its group inverse. Then

$$
a \circ a^{-1}=a \phi(\alpha \psi)^{-1}
$$

Now,

$$
\begin{aligned}
\left(a \circ a^{-1}\right) \circ\left(a \circ a^{-1}\right) & =\left(a \phi(a \psi)^{-1}\right) \phi \cdot\left(a \dot{\phi}(a \psi)^{-1}\right) \psi \\
& =a \phi^{2} \cdot(a \psi \dot{\psi})^{-1} \cdot a \dot{\phi} \psi \cdot\left(a \psi^{2}\right)^{-1} \\
& =a \phi(a \psi)^{-1} \\
& =a \circ a^{-1}
\end{aligned}
$$

so $(G, \circ)$ is $E$-inversive since $a$ was taken to be arbitrary in $G$. Let $e$ denote the identity element of $(G, \cdot)$. Since $(G, \circ)$ is stationary on the right it is rectangular, whence by Theorem 8 of [4], $G \circ G$ is the kernel of $G$ and

$$
G \circ G \cong H \times E
$$

where $E$ is the rectangular band consisting of the idempotents of $(G, \circ)$, and $H$ is the subgroup

$$
e \circ G \circ e=\{x \dot{\phi} \psi: x \in G\}
$$

of $(G, \circ)$. By [5] the mapping $\theta: G \rightarrow G \circ G$ defined by $\alpha \theta=\alpha \circ f$, where 
$f$ is the identity element of the maximal subgroup to which $a \circ a$ belongs, is onto, idempotent, and $a \theta \circ b \theta=a \circ b$ for all $a, b \in G$, whence $(G, \circ)$ is an inflation of $(G \circ G, \circ)$. Thus $(G, \circ)$ is an inflation of the direct product of a group and a rectangular band. (We note that $(H, \cdot)=(H, \circ)$.)

The structure of a semigroup $(G, \circ)$ generated by the monomial $x^{s} y^{t}$ is revealed by the following theorem, which is a consequence of Theorem 5.

Theorem 6. Let $(G, \circ)$ be a semigroup generated by the monomial $x^{s} y^{t}$ over the finite abelian group $(G, \cdot)$. Then $(G, \circ)$ is an inflation of its kernel $G \circ G$, and its kernel is isomorphic to the direct product of the subgroup

$$
H=\left\{x^{s t}: x \in G\right\}
$$

of $(G, \circ)$ and the rectangular band

$$
E=\left\{x \in G: x=x^{s+t}\right\} .
$$

Proof. Let $\phi, \psi$ be defined on $(G, \cdot)$ by $x \phi=x^{s}$ and $y \psi=y^{t}$. Then $\phi, \psi$ are endomorphisms of $(G, \cdot)$ since $(G, \cdot)$ is abelian. Also, $\phi^{2}=\phi$ and $\psi^{2}=\psi$ since $x^{s^{2}}=x^{s}$ and $x^{t^{2}}=x^{t}$ for all $x \in G$. Since

$$
\left(x^{s}\right)^{t}=x^{s t}=\left(x^{t}\right)^{s} \quad \text { for all } x \in G,
$$

it follows that $\phi$ and $\psi$ commute. Thus $\phi$ and $\psi$ as defined above satisfy the hypothesis of Theorem 5 , so $(G, \circ)$ is an inflation of its kernel $(G \circ G, \circ)$. Since $x \phi \psi=x^{s t}$ for $x \in G$, and since $x$ is an idempotent of $(G, \circ)$ if and only if $x^{s+t}=x$, it follows that

$$
G \circ G \cong H \times E
$$

where $H$ and $E$ are as defined in the statement of the theorem.

Let $(a, b)$ denote the greatest common divisor of integers $a$ and $b$. We have the following lemma concerning certain subgroups of a cyclic group.

Lemma 7. Let $G$ be a cyclic group of order $n$ with identity element $e$, and let $s$ be a nonnegative integer such that $n \mid s^{2}-s$. Then $G_{s-1}=\left\{x \in G: x^{s-1}=e\right\}$ is a subgroup of $G$ having order $(n, s-1)$.

Proof. It follows immediately that $G_{s-1}$ is a subgroup of $G$. Let $m$ denote the order of $G_{s-1}$, and let $d=(n, s-1)$. Since

$$
x^{s-1}=e=x^{n}, \quad \text { for all } x \in G_{s-1},
$$


it follows that $m \mid s-1$ and $m \mid n$, whence $m \leqq d$. Now, let $a$ be a generator of $G$. Then $a^{n / d}$ generates a subgroup $\left[a^{n / d}\right]$ of $G$, of order d. But $\left(a^{n / d}\right)^{s-1}=\left(a^{n}\right)^{(s-1) / d}=e$, so $a^{n / d} \in G_{s-1}$, whence $\left[a^{n / d}\right] \subseteq G_{s-1}$. Thus $d \leqq m$, and so $m=d=(n, s-1)$.

The next theorem gives the structure of the group $H$ in Theorem 6 , whenever $(G, \cdot)$ is a cyclic group.

THEOREM 7. If $(G, \circ)$ is a semigroup generated by the monomial $x^{s} y^{t}$ over the cyclic group $(G, \cdot)$ of order $n$, then $(G, \circ)$ is an inflation of its kernel $G \circ G$. Furthermore, its kernel is isomorphic to the direct product of the cyclic subgroup

$$
H=\left\{x^{s t}: x \in G\right\}
$$

of $(G, \circ)$ of order $(n$, st-1) and the rectangular band

$$
E=\left\{x \in G: x^{s+t}=x\right\} \text {. }
$$

Proof. Suppose $x^{s} y^{t}$ generates a semigroup over $(G, \cdot)$. Then the set $H$ defined above is the same as the set

$$
G_{s t-1}=\left\{x \in G: x^{s t-1}=e\right\} \text {. }
$$

Since $n \mid s^{2}-s$, and $n \mid t^{2}-t$, it follows that

$$
n \mid\left(s^{2}-s\right) t+s^{2}\left(t^{2}-t\right),
$$

whence $n \mid(s t)^{2}-s t$. By Lemma 7, $H$ has order $(n, s t-1)$. The remaining part of the proof follows immediately from Theorem 6 .

We conclude with a corollary to Theorem 7 which extends the results obtained in [3].

CoRollary 3. Let $\left(F_{q},+, \cdot\right)$ be a finite field of order $q$, and let $\left(F_{q}, \circ\right)$ be a semigroup generated by $x^{s} y^{t}$ over $\left(F_{q}, \cdot\right)$. Then $\left(F_{q}, \circ\right)$ is an inflation of the direct product of a cyclic group of order $(q-1$, st -1$)$, and a rectangular band, together with a zero element.

Proof. Let $F_{q}^{*}=F_{q} \backslash\{0\}$. Then $\left(F_{q}^{*}, \cdot\right)$ is the multiplicative group of $\left(F_{q},+, \cdot\right)$, hence is a cyclic group of order $q-1$. By Theorem $7,\left(F_{q}^{*}, \circ\right)$ is an inflation of the direct product of a cyclic group of order $(q-1, s t-1)$, and a rectangular band. Since

$$
F_{q}=F_{q}^{*} \cup\{0\}
$$

and 0 is a zero for $\circ$, the corollary holds. 


\section{REFERENCES}

1. A. H. Clifford and G. B. Preston, The Algebraic Theory of Semigroups, Vol. I, Mathematical Surveys 7, Amer. Math. Soc., Providence, R.I., 1961.

2. W. J. LeVeque, Topics in Number Theory, Vol. I, Addison-Wesley Publishing Co., Inc., Reading, Mass., 1956.

3. R. J. Plemmons and R. Yoshida, Generating polynomials for finite semigroups. To appear in Mathematishe Nachrichten.

4. G. Thierrin, Demi-groups inverse's et rectangulaires, Acad. Roy. Belg. Cl. Sci. (5) 41 (1955), 83-92, (MR 17, 10).

5. M. Yamada, $A$ note on middle unitary semigroups, Kōdai Math. Semi. Rep., 7 (1955), 49-52, (MR 17, 585).

Received October 23, 1970. This research is a portion of the author's doctoral dissertation written at the University of Tennessee under the direction of Dr. R. J. Plemmons.

UNIVERSITY OF TENNESSEE 


\title{
PACIFIC JOURNAL OF MATHEMATICS
}

\author{
EDITORS
}

\author{
H. SAMELSON \\ Stanford University \\ Stanford, California 94305 \\ C. R. HOBBY \\ University of Washington \\ Seattle, Washington 98105
}

J. DugundJI

Department of Mathematics

University of Southern California

Los Angeles, California 90007

RICHARD ARENS

University of California

Los Angeles, California 90024

\section{ASSOCIATE EDITORS}
E. F. BeCKenBaCH
B. H. NeUMANN
F. WOLF
K. Yoshida

\section{SUPPORTING INSTITUTIONS}

\author{
UNIVERSITY OF BRITISH COLUMBIA \\ CALIFORNIA INSTITUTE OF TECHNOLOGY \\ UNIVERSITY OF CALIFORNIA \\ MONTANA STATE UNIVERSITY \\ UNIVERSITY OF NEVADA \\ NEW MEXICO STATE UNIVERSITY \\ OREGON STATE UNIVERSITY \\ UNIVERSITY OF OREGON \\ OSAKA UNIVERSITY
}

\author{
UNIVERSITY OF SOUTHERN CALIFORNIA \\ STANFORD UNIVERSITY \\ UNIVERSITY OF TOKYO \\ UNIVERSITY OF UTAH \\ WASHINGTON STATE UNIVERSITY \\ UNIVERSITY OF WASHINGTON \\ AMERICAN MATHEMATICAL SOCIETY \\ NAVAL WEAPONS CENTER
}

The Supporting Institutions listed above contribute to the cost of publication of this Journal, but they are not owners or publishers and have no responsibility for its content or policies.

Mathematical papers intended for publication in the Pacific Journal of Mathematics should be in typed form or offset-reproduced, (not dittoed), double spaced with large margins. Underline Greek letters in red, German in green, and script in blue. The first paragraph or two must be capable of being used separately as a synopsis of the entire paper. The editorial "we" must not be used in the synopsis, and items of the bibliography should not be cited there unless absolutely necessary, in which case they must be identified by author and Journal, rather than by item number. Manuscripts, in duplicate if possible, may be sent to any one of the four editors. Please classify according to the scheme of Math. Rev. Index to Vol. 39. All other communications to the editors should be addressed to the managing editor, Richard Arens, University of California, Los Angeles, California, 90024.

50 reprints are provided free for each article; additional copies may be obtained at cost in multiples of 50 .

The Pacific Journal of Mathematics is published monthly. Effective with Volume 16 the price per volume (3 numbers) is $\$ 8.00$; single issues, $\$ 3.00$. Special price for current issues to individual faculty members of supporting institutions and to individual members of the American Mathematical Society: $\$ 4.00$ per volume; single issues $\$ 1.50$. Back numbers are available.

Subscriptions, orders for back numbers, and changes of address should be sent to Pacific Journal of Mathematics, 103 Highland Boulevard, Berkeley, California, 94708.

PUBLISHED BY PACIFIC JOURNAL OF MATHEMATICS, A NON-PROFIT CORPORATION

Printed at Kokusai Bunken Insatsusha (International Academic Printing Co., Ltd.), 270, 3chome Totsuka-cho, Shinjuku-ku, Tokyo 160, Japan. 


\section{Pacific Journal of Mathematics}

\section{Vol. 39, No. $3 \quad$ July, 1971}

William O'Bannon Alltop, 5-designs in affine spaces ................... 547

B. G. Basmaji, Real-valued characters of metacyclic groups ................. 553

Miroslav Benda, On saturated reduced products....................... 557

J. T. Borrego, Haskell Cohen and Esmond Ernest Devun, Uniquely representable semigroups. II.......................................

George Lee Cain Jr. and Mohammed Zuhair Zaki Nashed, Fixed points and stability for a sum of two operators in locally convex spaces ....................

Donald Richard Chalice, Restrictions of Banach function spaces ...............

Eugene Frank Cornelius, Jr., A generalization of separable groups ..............

Joel L. Cunningham, Primes in products of rings ......................

Robert Alan Morris, On the Brauer group of $Z$.

593

603

615

David Earl Dobbs, Amitsur cohomology of algebraic number rings ...............

Charles F. Dunkl and Donald Edward Ramirez, Fourier-Stieltjes transforms and

weakly almost periodic functionals for compact groups ...................

Hicham Fakhoury, Structures uniformes faibles sur une classe de cônes et

d'ensembles convexes ......................................

Leslie R. Fletcher, A note on C $\theta \theta$-groups.

Humphrey Sek-Ching Fong and Louis Sucheston, On the ratio ergodic theorem for

semi-groups............................................

James Arthur Gerhard, Subdirectly irreducible idempotent semigroups ...........

Thomas Eric Hall, Orthodox semigroups.....................

Marcel Herzog, $C \theta \theta$-groups involving no Suzuki groups ..........

669

687

John Walter Hinrichsen, Concerning web-like continua ..........

691

Frank Norris Huggins, A generalization of a theorem of F. Riesz.

695

Carlos Johnson, Jr., On certain poset and semilattice homomorphisms

703

Alan Leslie Lambert, Strictly cyclic operator algebras ...........

717

Howard Wilson Lambert, Planar surfaces in knot manifolds . . .

727

Robert Allen McCoy, Groups of homeomorphisms of normed linear spaces ....... 735

T. S. Nanjundiah, Refinements of Wallis's estimate and their generalizations ...... 745

Roger David Nussbaum, A geometric approach to the fixed point index .......... 751

John Emanuel de Pillis, Convexity properties of a generalized numerical range .... 767

Donald C. Ramsey, Generating monomials for finite semigroups ....

783

William T. Reid, A disconjugacy criterion for higher order linear vector differential equations...

Roger Allen Wiegand, Modules over universal regular rings...

Kung-Wei Yang, Compact functors in categories of non-archimedean Banach

spaces.

R. Grant Woods, Correction to: "Co-absolutes of remainders of Stone-Čech compactifications".

Ronald Owen Fulp, Correction to: "Tensor and torsion products of

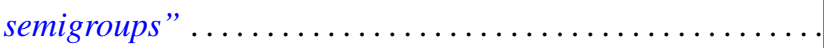

Bruce Alan Barnes, Correction to: "Banach algebras which are ideals in a banach 